\title{
Variable-delay feedback control of unstable steady states in retarded time-delayed systems
}

\author{
A. Gjurchinovsk* and V. Urumov \\ Institute of Physics, Faculty of Natural Sciences and Mathematics, \\ Sts. Cyril and Methodius University, P. O. Box 162, 1000 Skopje, Macedonia
}

(Dated: 24 December, 2009)

\begin{abstract}
We study the stability of unstable steady states in scalar retarded time-delayed systems subjected to a variable-delay feedback control. The important aspect of such a control problem is that timedelayed systems are already infinite-dimensional before the delayed feedback control is turned on. When the frequency of the modulation is large compared to the system's dynamics, the analytic approach consists of relating the stability properties of the resulting variable-delay system with those of an analogous distributed delay system. Otherwise, the stability domains are obtained by a numerical integration of the linearized variable-delay system. The analysis shows that the control domains are significantly larger than those in the usual time-delayed feedback control, and that the complexity of the domain structure depends on the form and the frequency of the delay modulation.
\end{abstract}

PACS numbers: $05.45 . \mathrm{Gg}, 02.30 . \mathrm{Ks}$

\section{INTRODUCTION}

In spite of the fact that the control problems have been thoroughly investigated from a theoretical aspect and the results are being implemented in concrete real systems for several decades [1], the control of chaotic dynamical systems is a relatively new area of research. The appearance of the pioneering paper by Ott, Grebogy and Yorke (OGY) in 1990 boosted quite an interest among nonlinear scientists [2], being a reason for a large number of published papers on chaos control [3 6]. The OGY method utilizes the existence of infinitely many unstable periodic orbits (UPO) within the structure of the chaotic attractor, applying a small externally controlled perturbation to suitably chosen parameters of the system when the trajectory is in the neighborhood of an UPO whose control is desirable. The system is then externally forced to follow otherwise unstable behavior corresponding to that UPO. The numerical simulations and the experimental implementations showed that the method by itself has some drawbacks concerning the robustness with respect to the external noise and its practical realization, since it requires a continuous monitoring of the evolution of the system from the outside and the knowledge of the equations that describe the system's dynamics.

The OGY idea stimulated a development of a rich variety of new chaos control techniques. Among those is the time-delayed feedback control (TDFC) proposed by Pyragas in 1992 [7, 8], shown to be much more flexible for practical purposes with respect to OGY (the monitoring of the system and the knowledge of the exact positions of UPOs are not required) and quite robust against the effects of noise. The control force is applied as a continuous feedback proportional to the difference between the current state of the system and the state of the sys-

*Electronic address: agjurcin@pmf.ukim.mk tem delayed by the constant time $T$. If the time delay $T$ is chosen to coincide with an integer multiple of the period of the target UPO, then the control force will vanish when the target state is reached and the control is noninvasive. For stabilization of unstable steady states (USS), the choice of the parameter $T$ is not as restrictive as in the case of UPOs, and the interval of $T$ for which TDFC is successful is shown to be system-dependent [9 11].

In parallel to various practical applications of TDFC 12 21], an effort has been put into progress to generalize or modify the original control scheme in order to improve its performance. Some extended TDFC schemes employ multiple time-delays to stabilize strongly unstable periodic orbits [22 25]. Others are introducing an oscillating feedback gain [26] or an extra unstable degree of freedom in the feedback loop 27-29] to overcome the so-called odd-number limitation [30-32], which was refuted recently 33 36. In a recent work [37], it has been shown that the efficiency of the TDFC method to control USS can be significantly improved by including a variable time-delay into the TDFC scheme in a form of a deterministic or stochastic modulation in a fixed interval around a nominal delay value. Stochastic changes in the delay time are natural due to the omnipresent noise in any physical system. In the circumstances, the enhancement of noise along the delay line could be desirable as it is leading to improved stability of the system. On the other hand the modulated delay described by some periodic function could be realized by periodically changing some characteristic distances in electric or laser systems by introducing piezoelements. This variable delay feedback control (VDFC) has been shown successful in stabilization of USS in low-dimensional chaotic systems using different types of delay modulations. The ongoing analysis shows that VDFC can also improve the control domain of UPOs with respect to TDFC for a specific choice of the delay modulation 38.

The purpose of this paper is to investigate the effects of stabilization of unstable equilibria by a variable-delay 
feedback control in a class of nonlinear dynamical systems described by scalar retarded delay-differential equations (RDDE) involving the value of the state variable at a discrete time lag. A delay differential equation is called retarded if the highest order derivative only occurs with one value of the argument, and this argument is not less than the arguments of the unknown function and its lower order derivatives appearing in the equation [39 41]. In contrast to low dimensional dynamical systems, delay differential equations are infinite dimensional, since it is necessary to specify a continuum of initial conditions over the interval length equal to the time delay. The interest for such equations is caused by their frequent occurrence in numerous physical, biological and engineering models, where the time delays are a natural manifestation of the system's dynamics [42 45].

The paper is organized as follows. In Section II we perform a linear stability analysis of USS in the free running RDDE system and in the system under VDFC. The frequency of the delay modulation in the feedback loop is considered to be sufficiently large compared to the intrinsic timescale of the unperturbed system, allowing an approximation of the variable delay system with a distributed delay system [46]. In Section III, we numerically illustrate the VDFC method in the chaotic Mackey-Glass system. The domains of successful control are first computed for high-frequency modulations of the time delay for different values of the modulation amplitude. The planes of the control domains are parametrized by the feedback gain and the nominal delay of the control force for a fixed delay of the RDDE, and also, by the time delay of the original system and the nominal delay of the feedback control force for a fixed value of the feedback gain. The control domains are also determined for a lowfrequency modulation in the plane of the feedback gain and the nominal delay of the control force for different values of the frequency of the modulation. The results show a significant enlargement of stability areas of VDFC with respect to TDFC within a certain range of the control parameters, sometimes resulting in a complicated reconfiguration depending on the type, the amplitude and the frequency of the delay modulation. The conclusions are summarized in Section IV.

\section{STABILITY ANALYSIS}

We consider a general nonlinear dynamical system described by a scalar autonomous RDDE in the form:

$$
\dot{x}(t)=F\left[x(t), x\left(t-T_{1}\right)\right],
$$

where $T_{1} \geq 0$ is a constant delay time, and $F$ is an arbitrary nonlinear function of the state variable $x$, having a past dependence through the same state variable $x$ but at $T_{1}$ time units in the past. The presence of the delay term $x\left(t-T_{1}\right)$ is a cause for the system (1) to be infinite dimensional, since a continuum of initial conditions over the time interval $\left[-T_{1}, 0\right]$ is required in order to uniquely specify the future behavior of the system. The system possesses a set of fixed points $\left\{x_{i}^{*}\right\}$ that are solution of $F\left[x^{*}(t), x^{*}\left(t-T_{1}\right)\right]=0$, and the stability of a particular fixed point $x^{*}$ can be obtained by linearizing Eq. (1) in the vicinity of $x^{*}$. The linearized version of (1) around $x^{*}$ has a general form:

$$
\dot{\tilde{x}}(t)=A \widetilde{x}(t)+B \widetilde{x}\left(t-T_{1}\right),
$$

where $A$ and $B$ are real constants. We made a coordinate transformation from $x$ to $\widetilde{x}$ according to $\widetilde{x}(t)=x(t)-x^{*}$ such that the fixed point is at the origin as expressed in the new coordinate. Employing the usual ansatz $x(t) \sim$ $\exp (\lambda t)$ in (2) we obtain the characteristic equation:

$$
\lambda=A+B e^{-\lambda T_{1}} .
$$

This is a transcendental equation in $\lambda$, possessing a countable infinite set of complex solutions $\left\{\lambda_{i}\right\}$ defining the eigenvalues of the fixed point at the origin. The origin is stable if and only if each $\lambda_{i}$ has a negative real part, it is unstable if at least one $\lambda_{i}$ has a positive real part, and it is marginally unstable if the largest real part of all the eigenvalues $\left\{\lambda_{i}\right\}$ is zero.

The goal of this paper is to investigate the possibility of stabilization of the unstable fixed point $x^{*}$ of the system (11) by applying a Pyragas-type feedback force $u(t)$ with a variable time delay [37]:

$$
\begin{aligned}
u(t) & =K[x(t-\tau(t))-x(t)], \\
\tau(t) & =T_{2}+\varepsilon f(\nu t),
\end{aligned}
$$

such that for a given set of control parameters $\left\{K, T_{2}, \varepsilon, \nu\right\}$ the unstable fixed point $x^{*}$ of the unperturbed system (10) becomes stable in the presence of the feedback term (4). The control parameter $K$ is the feedback gain characterizing the strength of the feedback, and $\tau(t)$ is the variable time delay. We will consider a variation in a form of a deterministic modulation around a nominal delay value described by the control parameter $T_{2}$. We take the delay function $f: \mathbb{R} \rightarrow[-1,1]$ to be periodic with zero mean, with $\varepsilon$ and $\nu$ being the parameters determining the amplitude and the frequency of the modulation, respectively. The form of the control force (4)-(5) implies that since $\tau(t) \geq 0$, the values of the amplitude $\varepsilon$ are restricted to the interval $\left[0, T_{2}\right]$. In the presence of the control force (4), the system (10) has the form:

$$
\dot{x}(t)=F\left[x(t), x\left(t-T_{1}\right)\right]+u(t),
$$

and the linearized version around $x^{*}$ in terms of the new coordinate $\widetilde{x}$ is:

$$
\dot{\tilde{x}}(t)=A \widetilde{x}(t)+B \widetilde{x}\left(t-T_{1}\right)+\widetilde{u}(t),
$$

where

$$
\widetilde{u}(t)=K[\widetilde{x}(t-\tau(t))-\widetilde{x}(t)] .
$$


The stability of the origin can be inferred by numerically integrating the linear variable-delay system (7)-(8) for different values of $K, T_{2}, \varepsilon$ and $\nu$, thus determining the domains in the $\left(K, T_{2}, \varepsilon, \nu\right)$ hyperspace for which the stabilization becomes possible.

For a sufficiently large variation of the time delay $\tau(t)$, the stability of the linear variable-delay system (7)-(8) becomes amenable for analytical treatment [46]. From the stability point of view, if the frequency of the delay variation $\nu$ is sufficiently large, then the linear system (7) with a variable time-delay (8) behaves as the following time-invariant system with a distributed delay (Theorem A1, Appendix A):

$$
\begin{aligned}
\dot{\widetilde{x}}(t)= & A \widetilde{x}(t)+B \widetilde{x}\left(t-T_{1}\right)+ \\
& K\left(\int_{-1}^{1} w(\eta) \widetilde{x}\left(\varepsilon \eta+t-T_{2}\right) d \eta-\widetilde{x}(t)\right),
\end{aligned}
$$

with $w$ being the weight related to the probability distribution of the delay function $f$ in the interval of its periodicity, satisfying $\int_{-1}^{1} w(\eta) d \eta=1$ (see Table I). The stability of the distributed delay system (9) is determined by the roots $\lambda_{i}$ of its characteristic equation:

$$
\lambda=A+B e^{-\lambda T_{1}}+K\left[e^{-\lambda T_{2}} g(\lambda \varepsilon)-1\right],
$$

where $g: \mathbb{C} \rightarrow \mathbb{C}$ is a smooth complex function defined as:

$$
g(\lambda \epsilon)=\int_{-1}^{1} w(\eta) e^{\lambda \epsilon \eta} d \eta
$$

In this sense, the solutions $\left\{\lambda_{i}\right\}$ determining the stability of the comparison system (9) can be considered as effective eigenvalues describing the overall stability of the original variable delay system (7)-(8), providing that the delay frequency $\nu$ is large compared to the system's dynamics. Numerical simulations showed that the threshold for the frequency $\nu$ above which this type of comparative analysis becomes valid needs not to be very high, and that its value depends on the actual system under investigation.

\section{A. Stability of the unperturbed system}

In the absence of control, the stability of the fixed point $x^{*}$ is determined by the roots of the characteristic equation (3). Let $H_{0}$ be a function of $\lambda$ defined as:

$$
H_{0}(\lambda)=\lambda-A-B e^{-\lambda T_{1}} .
$$

With the aid of this characteristic quasipolinomial $H_{0}(\lambda)$, Eq. (3) can be written as $H_{0}(\lambda)=0$. We would like to find the range of the values for $A, B$ and $T_{1}$ for which $x^{*}$ is stable.

Since $H_{0}(\lambda)$ is a smooth function on $\lambda$, it is useful to consider the behavior of $H_{0}(\lambda)$ as $\lambda$ changes continuously over the real interval $[0,+\infty)$. Specifically, at the ends of this interval, we have:

$$
\begin{aligned}
\lim _{\lambda \rightarrow \infty} H_{0}(\lambda) & =+\infty, \\
\lim _{\lambda \rightarrow 0^{+}} H_{0}(\lambda) & =-(A+B) .
\end{aligned}
$$

If $A+B>0$, then $H_{0}$ changes its sign at least once as $\lambda$ sweeps along the positive real axis. Consequently, there exists at least one positive real root of the characteristic equation $H_{0}(\lambda)=0$, rendering the fixed point unstable for any $T_{1}$. If $A+B=0$, then $\lambda=0$ is a root of the characteristic equation (3), and the fixed point is unstable, or at least marginally unstable. Hence, a necessary (but not sufficient!) condition for stability of the fixed point is:

$$
A+B<0
$$

Taking into account that the boundary between stability and instability (the threshold of control) occurs when the maximal value from all the real parts in the set of solutions $\left\{\lambda_{i}\right\}$ is zero, we look for a solution of Eq. (3) in the form $\lambda=i \omega, \omega \in \mathbb{R}$, and separate real and imaginary parts of the resulting equation to obtain:

$$
\begin{aligned}
-A & =B \cos \left(\omega T_{1}\right), \\
-\omega & =B \sin \left(\omega T_{1}\right) .
\end{aligned}
$$

[We stress that a zero on the imaginary axis for some set of parameters $A, B$ and $T_{1}$ does not necessarily mean that all the other zeros of the characteristic polinomial $H_{0}(\lambda)$ for the same set of parameters have negative real parts. The stability boundary is just one set of solutions of Eqs. (16) -(17).] By eliminating the trigonometric terms from the last pair of equations, we get:

$$
\omega^{2}=B^{2}-A^{2}
$$

from which we conclude that Eq. (3) can have a solution for $\lambda$ on the imaginary axis if and only if $|B|>|A|$. Taking into account that $T_{1}>0$, from Eq. (16) we obtain:

$$
T_{1}=\frac{\operatorname{Arccos}(-A / B)+2 n \pi}{\sqrt{B^{2}-A^{2}}},
$$

where $n$ is a nonnegative integer, and Arccos denotes the principal value of the arccosine function. Obviously, the first value of $T_{1}$ for which $H_{0}(\lambda)=0$ has a solution for $\lambda$ on the imaginary axis is:

$$
T_{1}^{*}=\frac{\operatorname{Arccos}(-A / B)}{\sqrt{B^{2}-A^{2}}},
$$

which follows from Eq. (19) by setting $n=0$. The behavior of the real part of $\lambda$ at the values for $T_{1}$ in Eq. (19) is determined by the derivative $d \lambda / d T_{1}$ at $\lambda=i \omega$. By implicit differentiation of Eq. (3) with respect to $T_{1}$, we obtain:

$$
\frac{d \lambda}{d T_{1}}=-\frac{\lambda B e^{-\lambda T_{1}}}{1+B T_{1} e^{-\lambda T_{1}}}=-\frac{\lambda(\lambda-A)}{1+T_{1}(\lambda-A)},
$$


TABLE I: A representation of the delay function $f$, the weight $w$ of the distributed delay system, and the function $g$, corresponding to three different types of delay modulations. By $I_{0}$ we denote the modified Bessel function of the first kind of order zero, $J_{0}$ is the Bessel function of the first kind of order zero, and $\delta$ is the Dirac delta function.

\begin{tabular}{c|c|c|c|c}
\hline \hline Type & $f(t)$ & $w(t)$ & $g(\lambda \varepsilon)$ & $g(i \omega \varepsilon)$ \\
\hline \hline Sawtooth wave & $\left\{\begin{array}{c|c}\frac{2}{\pi}\left(t-\frac{\pi}{2}\right), \quad t \in[0, \pi) \\
\frac{2}{\pi}\left(\frac{3 \pi}{2}-t\right), t \in[\pi, 2 \pi)\end{array}\right.$ & $\frac{1}{2}$ & $\frac{\sinh (\lambda \varepsilon)}{\lambda \varepsilon}$ & $\frac{\sin (\omega \varepsilon)}{\omega \varepsilon}$ \\
\hline Sine wave & $\left\{\begin{array}{c}-1, \quad t \in[0, \pi) \\
1, \quad t \in[\pi, 2 \pi)\end{array}\right.$ & $\frac{\delta(t-1)+\delta(t+1)}{\pi \sqrt{1-t^{2}}}$ & $\cosh (\lambda \varepsilon)$ & $\cos (\omega \varepsilon)$ \\
\hline Square wave & $\left\{\begin{array}{c}2 \\
-1, \lambda \varepsilon)\end{array}\right.$ & $J_{0}(\omega \varepsilon)$ \\
\hline \hline
\end{tabular}

from which at $\lambda=i \omega$ we get:

$$
\operatorname{Re}\left(\frac{d \lambda}{d T_{1}}\right)_{\lambda=i \omega}=\frac{\omega^{2}}{\left(1-A T_{1}\right)^{2}+\left(\omega T_{1}\right)^{2}} .
$$

Since the sign of this derivative is always positive, the sign of the real part of $\lambda$ switches from negative to positive when the zero of the characteristic quasipolinomial $H_{0}(\lambda)$ crosses the imaginary axis. On the other hand, as an implication of the Rouché theorem, the number of roots (counting multiplicity) on the complex right half plane (RHP) and the number of roots on the complex left half plane (LHP) can be changed (or, more correctly, interchanged) only if a zero appears on or crosses the imaginary axis [47, 48]. As a consequence, in the case under consideration $|B|>|A|$, all the zeros of the characteristic quasipolinomial $H_{0}(\lambda)$ lie on the LHP if $T_{1}$ is in the interval $\left[0, T_{1}^{*}\right)$ providing that all the zeros were on the LHP before the first crossing of the imaginary axis has occured. However, this is evidently not true for other intervals separated by the corresponding values of $T_{1}$ given by Eq. (19) for $n>0$, since the first zerocrossing of the imaginary axis occurs for $T_{1}=T_{1}^{*}$, and according to Eq. (22) every crossing is from the LHP to the RHP.

In the case $A \geq 0$, the necessary condition for the stability of the fixed point $x^{*}$ is $B<-A$ [see Eq. (15)], which is an interval of $B$ that belongs to the range $|B|>|A|$ for which the characteristic equation (3) can have a solution on the imaginary axis. From the previous discussion, the possibility for all the zeros of the quasipolinomial $H_{0}(\lambda)$ to lie on the LHP necessary imply $T_{1} \in\left[0, T_{1}^{*}\right)$. Since for $T_{1}=0$ the characteristic equation (3) is reduced to $\lambda=A+B<0$, and since the crossing of the imaginary axis occurs for $T_{1}=T_{1}^{*}$, we conclude that all the zeros $\left\{\lambda_{i}\right\}$ have negative real parts in this case if and only if $B<-A$ and $T_{1} \in\left[0, T_{1}^{*}\right)$.
In the case $A<0$, the necessary condition for the stability of the fixed point is $B<|A|$. In the subinterval $B \in[-|A|,|A|)$, the characteristic quasipolinomial (12) cannot have a zero on the imaginary axis. Choosing $B=$ 0 , from (3) we have $\lambda=A<0$. Since crossing of the imaginary axis does not occur for this subinterval of $B$, it follows that all the zeros $\left\{\lambda_{i}\right\}$ for $B \in[-|A|,|A|)$ lie on the LHP for any $T_{1}>0$. On the other hand, in the range $B<-|A|$ the characteristic quasipolinomial (12) can have a zero on the imaginary axis. Putting $T_{1}=0$ in (3) we obtain $\lambda=A+B<0$, which means that when $B<-|A|$ all the zeros $\left\{\lambda_{i}\right\}$ have negative real parts when $T_{1} \in\left[0, T_{1}^{*}\right)$.

The results are summarized with the following theorem:

Theorem 1 Let the linear RDDE:

$$
\dot{\tilde{x}}(t)=A \widetilde{x}(t)+B \widetilde{x}\left(t-T_{1}\right), \quad A, B \in \mathbb{R}
$$

be a result of linearization of a corresponding nonlinear $R D D E$ with a constant delay $T_{1}$ :

$$
\dot{x}(t)=F\left[x(t), x\left(t-T_{1}\right)\right]
$$

around some fixed point $x^{*}$ of the latter expressed in coordinates in which the fixed point is at the origin. Furthermore, let $T_{1}^{*}>0$ be a real positive constant defined as:

$$
T_{1}^{*}=\frac{\operatorname{Arccos}(-A / B)}{\sqrt{B^{2}-A^{2}}} .
$$

Then, the fixed point $x^{*}$ is locally asymptotically stable in each of the following cases:

(a). $B<-|A|$ and $T_{1} \in\left[0, T_{1}^{*}\right)$; 
(b). $B \in[-|A|,|A|), A<0$ and $T_{1}>0$.

Otherwise, $x^{*}$ is unstable.

\section{B. Stability under variable-delay feedback control (high-frequency modulation)}

In the following, we consider the modulation frequency $\nu$ to be above the threshold, allowing an analysis of the variable delay system (7)-(8) as a distributed delay system (9). When the control is switched on, the stability of the fixed point $x^{*}$ is determined by the roots $\left\{\lambda_{i}\right\}$ of the characteristic equation (10). If we define:

$$
H_{\varepsilon}(\lambda)=\lambda-A-B e^{-\lambda T_{1}}+K\left[1-e^{-\lambda T_{2}} g(\lambda \varepsilon)\right],
$$

then Eq. (10) can be rewritten as $H_{\varepsilon}(\lambda)=0$. Assuming that in the absence of control, the parameters $A, B$ and $T_{1}$ of the unperturbed system are such that $x^{*}$ is unstable, we look for the values of the control parameters $K, T_{2}$ and $\varepsilon$ for which the fixed point is stabilized. In other words, we would like to find the set of points (i. e. to determine the domain of control) in the parameter space $\left(K, T_{2}, \varepsilon\right)$ for which all the zeros of the characteristic quasipolynomial $H_{\varepsilon}(\lambda)$ lie on the LHP, while, at the same time, the characteristic quasipolynomial $H_{0}(\lambda)$ of the unperturbed system has at least one zero in the RHP.

Before we proceed with the analytical description of the control boundaries, it is interesting to consider the behavior of $H_{\varepsilon}(\lambda)$ as $\lambda$ changes continuously over the positive real axis. Taking into account that $g(0)=$ $\int_{-1}^{1} w(\eta) d \eta=1$, from Eq. (23) we obtain:

$$
\begin{aligned}
\lim _{\lambda \rightarrow \infty} H_{\varepsilon}(\lambda) & =+\infty, \\
\lim _{\lambda \rightarrow 0^{+}} H_{\varepsilon}(\lambda) & =-(A+B),
\end{aligned}
$$

which coincide with the limits (13)-(14) for the characteristic polynomial $H_{0}(\lambda)$ of the unperturbed system, leading to the same necessary condition (15) for stability of the fixed point. Since (15) does not include the dependence on the control parameters $K, T_{2}$ and $\varepsilon$, we conclude that VDFC is unsuccessful for any values of the control parameters if the linearized version (2) of the unperturbed system around $x^{*}$ is such that $A+B>0$. This important result is expressed in the following theorem.

Theorem 2 Let $\dot{\widetilde{x}}(t)=A \widetilde{x}(t)+B \widetilde{x}\left(t-T_{1}\right), A, B \in \mathbb{R}$, be a linearization around the fixed point $x^{*}$ of the corresponding nonlinear RDDE with a constant delay $T_{1}$. If $A+B>0$, then the variable-delay feedback control (4)(5) cannot stabilize the unstable fixed point $x^{*}$ for any value of the control parameters $K, T_{2}$ and $\varepsilon$.

The limitation of the VDFC method imposed by Theorem 2 is a kind of an analogue to the odd-number limitation $30-32$ in the case of delayed feedback control of systems described by ordinary differential equations, whose validity was recently refuted [33 36] for the case of unstable periodic orbits.

Exact analytical description of the domains of successful control in the parameter space $\left(K, T_{2}, \varepsilon\right)$ is difficult for the characteristic Eq. (10) due to the complexity of the terms involving the dependence on $\lambda$. Thus, one should solve Eq. (10) numerically in order to calculate the control domains. To this extend, it is possible to obtain expressions for the parametric representation of the control boundaries parametrized by a Hopf frequency $\omega$. Substituting $\lambda=i \omega$ in Eq. (10) and separating real and imaginary parts, we obtain:

$$
\begin{aligned}
K g(i \omega \varepsilon) \cos \omega T_{2} & =K-A-B \cos \omega T_{1}, \\
K g(i \omega \varepsilon) \sin \omega T_{2} & =-\omega-B \sin \omega T_{1} .
\end{aligned}
$$

Elimination of $T_{2}$ from the last pair of equation yields a quadratic equation in $K$ :

$$
\begin{aligned}
& {\left[1-g(i \omega \varepsilon)^{2}\right] K^{2}-2\left(A+B \cos \omega T_{1}\right) K} \\
& \quad+\left(A+B \cos \omega T_{1}\right)^{2}+\left(\omega+B \sin \omega T_{1}\right)^{2}=0
\end{aligned}
$$

which can be solved for $K$ in terms of $\omega$ to get:

$$
\begin{aligned}
K(\omega) & =\frac{A+B \cos \omega T_{1}}{1-[g(i \omega \varepsilon)]^{2}} \pm \frac{1}{1-[g(i \omega \varepsilon)]^{2}} \\
& \times\left[[g(i \omega \varepsilon)]^{2}\left(A+B \cos \omega T_{1}\right)^{2}\right. \\
& \left.+\left([g(i \omega \varepsilon)]^{2}-1\right)\left(\omega+B \sin \omega T_{1}\right)^{2}\right]^{1 / 2}
\end{aligned}
$$

On the other hand, by dividing (27) and (26), we obtain:

$$
T_{2}(\omega)=\frac{1}{\omega}\left[\operatorname{Arctan}\left(\frac{-\omega-B \sin \omega T_{1}}{K-A-B \cos \omega T_{1}}\right) \pm n \pi\right]
$$

which, together with Eq. (29), describe the stability boundary for a fixed $\varepsilon$ in the $\left(K, T_{2}\right)$ plane, parametrized by $\omega$.

It is also useful to study the stability boundaries of the controlled system for a fixed feedback strength $K$ and modulation amplitude $\varepsilon$ in the parameter plane of the two delay times $\left(T_{1}, T_{2}\right)$. Following the idea in Ref. [48], we rewrite Eq. (10) as:

$$
1+a(\lambda) e^{-\lambda T_{1}}+b(\lambda) e^{-\lambda T_{2}}=0
$$

where $a(\lambda)$ and $b(\lambda)$ are given by:

$$
\begin{aligned}
& a(\lambda)=\frac{B}{A-K-\lambda}, \\
& b(\lambda)=\frac{K g(\lambda \varepsilon)}{A-K-\lambda} .
\end{aligned}
$$

At the control boundary $(\lambda=i \omega)$ the three terms in Eq. (31) can be considered as three vectors in the complex plane, with the corresponding magnitudes $1,|a(i \omega)|$ and $|b(i \omega)|$. According to Eq. (31), the sum of these vectors is a zero vector, thus forming the triangle shown in Fig. 


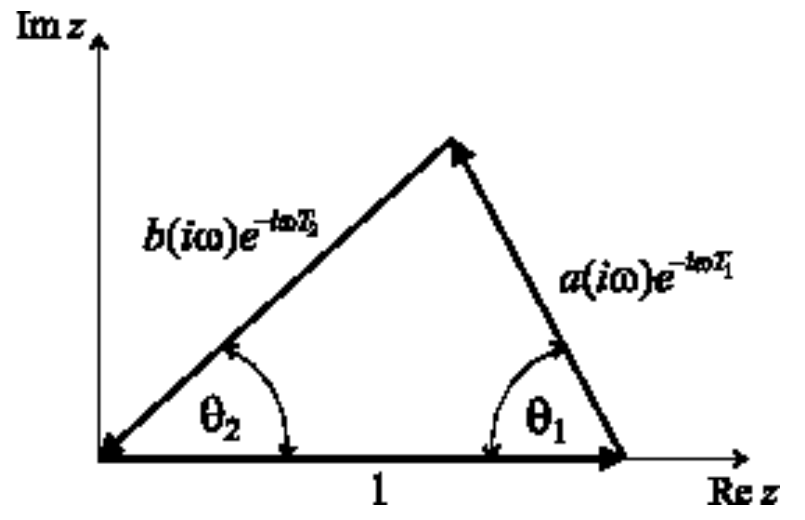

FIG. 1: Diagram in the complex plane $z$, associated with the derivation of the parametric representation of the stability boundary in $\left(T_{1}, T_{2}\right)$ plane.

1. From Fig. 1, it is straightforward to obtain the parametric representation of $T_{1}$ and $T_{2}$ on the Hopf frequency $\omega$ :

$$
\begin{gathered}
T_{1}(\omega)=\frac{\operatorname{Arg}[a(i \omega)]+(2 u-1) \pi \pm \theta_{1}}{\omega} \geq 0, \\
u=u_{0}^{ \pm}, u_{0}^{ \pm}+1, u_{0}^{ \pm}+2 \ldots, \\
T_{2}(\omega)=\frac{\operatorname{Arg}\left[b(i \omega]+(2 v-1) \pi \mp \theta_{2}\right.}{\omega} \geq 0, \\
v=v_{0}^{ \pm}, v_{0}^{ \pm}+1, v_{0}^{ \pm}+2 \ldots,,
\end{gathered}
$$

where $u_{0}^{ \pm}$and $v_{0}^{ \pm}$are the smallest possible integers such that the corresponding values of $T_{1}$ and $T_{2}$ are all nonnegative, and $\theta_{1}, \theta_{2} \in[0, \pi]$ are the internal angles of the triangle shown in Fig. 1 calculated from the law of cosines as:

$$
\begin{aligned}
& \theta_{1}=\operatorname{Arccos}\left(\frac{1+|a(i \omega)|^{2}-|b(i \omega)|^{2}}{2|a(i \omega)|}\right), \\
& \theta_{2}=\operatorname{Arccos}\left(\frac{1+|b(i \omega)|^{2}-|a(i \omega)|^{2}}{2|b(i \omega)|}\right) .
\end{aligned}
$$

In the case when the nominal delay $T_{2}$ of the feedback control force coincides with the delay of the original system $T_{1}$, the characteristic Eq. (10) is reduced to:

$$
\lambda-A+K-[B+K g(\lambda \varepsilon)] e^{-\lambda T}=0,
$$

where we use $T=T_{1}=T_{2}$. At the stability boundary $(\lambda=i \omega)$ the last complex equation can be represented as a pair of two real equations:

$$
\begin{aligned}
& {[B+K g(i \omega \varepsilon)] \cos \omega T=K-A,} \\
& {[B+K g(i \omega \varepsilon)] \sin \omega T=-\omega,}
\end{aligned}
$$

which can be manipulated to obtain a parametric representation of the control boundary in the $(K, T)$ plane in terms of $\omega$ :

$$
\begin{aligned}
K(\omega)= & \frac{A+B g(i \omega \varepsilon)}{1-[g(i \omega \varepsilon)]^{2}} \pm \frac{1}{1-[g(i \omega \varepsilon)]^{2}}\left[(A+B g(i \omega \varepsilon))^{2}\right. \\
& \left.+\left([g(i \omega \varepsilon)]^{2}-1\right)\left(A^{2}+B^{2}-\omega^{2}\right)\right]^{1 / 2} \\
T(\omega)= & \frac{1}{\omega}\left[\operatorname{Arctan}\left(\frac{-\omega}{K-A}\right) \pm n \pi\right] .
\end{aligned}
$$

When $\varepsilon=0$, VDFC reduces to the usual Pyragas control scheme (TDFC) with a constant delay $T_{2}$. Since TDFC is a special case of VDFC when the modulation of the control delay in the feedback force is absent, the parametric representations of the control boundaries for TDFC simply follow from the ones derived in the case of VDFC by letting $\varepsilon=0$ (or, equivalently, $g(0)=1$ ) in the corresponding equations. For example, from Eqs. (26)(27) with $\varepsilon=0$ we obtain the parametric representation of the TDFC boundary in the $\left(K, T_{2}\right)$ plane parametrized by $\omega$ :

$$
\begin{array}{r}
K(\omega)=\frac{\left(A+B \cos \omega T_{1}\right)^{2}+\left(\omega+B \sin \omega T_{1}\right)^{2}}{2\left(A+B \cos \omega T_{1}\right)}, \\
T_{2}(\omega)=\frac{1}{\omega}\left[\operatorname{Arctan}\left(\frac{-\omega-B \sin \omega T_{1}}{K-A-B \cos \omega T_{1}}\right) \pm n \pi\right] .
\end{array}
$$

It is interesting to note that when $T_{1}=T_{2}=T$ in the case of TDFC, the corresponding characteristic equation can be written as:

$$
\lambda=A^{\prime}+B^{\prime} e^{-\lambda T},
$$

where $A^{\prime}=A-K$ and $B^{\prime}=B+K$. Noting the equivalency between Eq. (45) and Eq. (3), the exact analytical description of the stability domain in this case immediately follows from Theorem 1 .

\section{NUMERICAL EXAMPLE}

To test the VDFC method for stabilization of unstable steady states in chaotic RDDE systems, we will use the paradigmatic Mackey-Glass system introduced as a model for regeneration of blood cells in patients with leukemia [49 53]. The Mackey-Glass equation in the presence of VDFC states:

$$
\dot{x}(t)=\frac{a x\left(t-T_{1}\right)}{1+\left[x\left(t-T_{1}\right)\right]^{c}}-b x(t)+u(t),
$$

where $u(t)$ is given by Eqs. (4)-(5). Here $x(t)$ is a concentration of circulating blood cells, and $a, b$ and $c$ are parameters of the free running system, involved in the description of the dependence of the production/destruction of the blood cells as a function of $x(t)$ and $x\left(t-T_{1}\right)$, respectively. We will consider the typical values $a=0.2, b=0.1$ and $c=10$. 




FIG. 2: Representative samples of phase plots $x(t)$ vs. $x\left(t-T_{1}\right)$ for the uncontrolled Mackey-Glass system at different values of $T_{1}$ : (a) $T_{1}=4$ - the trajectory is attracted to the stable equilibrium point $x_{3}^{*}=-1$; (b) $T_{1}=8$ - the trajectory approaches a limit cycle; (c) $T_{1}=15$ - the attractor has evolved into a period-2 cycle; (d) $T_{1}=23$ - chaos. The simulations were performed using the MATLAB routine dde23 for integrating delay-differential equations with constant delays.

In the absence of control $[u(t)=0]$, the system (46) has a set of three fixed points $x_{1}^{*}=0, x_{2}^{*}=+1$ and $x_{3}^{*}=-1$ being solutions of:

$$
\frac{a x^{*}}{1+x^{* c}}-b x^{*}=0
$$

The stability of each $x_{i}^{*}$ is obtained by linearizing the unperturbed system around $x_{i}^{*}$, leading to Eq. (2) with:

$$
A=-b, \quad B=a \frac{1+(1-c) x^{* c}}{\left(1+x^{* c}\right)^{2}}
$$

and the corresponding characteristic equation is given by Eq. (3). For $x_{1}^{*}=0$, we have $A=-b=-0.1$ and $B=$ $a=0.2$. Using Theorem 1 we deduce that the fixed point $x_{1}^{*}$ is unstable for any $T_{1}$. For $x_{2,3}^{*}= \pm 1$, we have $A=$ $-b=-0.1$ and $B=a(2-c) / 4=-0.4$, indicating that this pair of fixed points are characterized by the same type of stability. From Theorem 1 we conclude that $x_{2,3}^{*}$ are stable if and only if $T_{1} \in[0,4.7082)$. Figure 2 shows the trajectory of the unperturbed system in $x(t)$ vs. $x(t-$ $T_{1}$ ) coordinate space for four different values of $T_{1}$. Panel (a) shows the evolution of the system for $T_{1}=4$. Since for this value of $T_{1}$ the fixed points $x_{2,3}^{*}$ are stable, the preference of the system towards $x_{2}^{*}=+1$ or $x_{3}^{*}=-1$ depends on the initial conditions. Panels (b)-(d) in Fig. 2 correspond to $T_{1}=8,15$ and 23 , respectively, showing the growth of the limit cycle through a period-doubling bifurcation sequence, and the eventual appearance of a chaotic attractor.
In performing the stability analysis under VDFC, we will first consider a high-frequency modulation of the control delay $\tau(t)$. The limitation imposed by Theorem 2 asserts that the fixed point $x_{1}^{*}$ cannot be stabilized with VDFC for any values of the control parameters $K, T_{2}$ and $\varepsilon$. The validity of this assertion has been verified by the numerical simulations, showing the absence of the domains of successful control in the corresponding parametric planes. On the other hand, the stability of the fixed points $x_{2,3}^{*}= \pm 1$ is determined by the roots $\left\{\lambda_{i}\right\}$ of the characteristic Eq. (10) with $A$ and $B$ given by Eq. (48). Even though there exists an infinite number of roots $\lambda_{i}$ of Eq. (10), only a finite number of them have real parts greater than a given constant. A computation of the rightmost characteristic roots with large enough accuracy is a nontrivial nonlinear eigenvalue problem, and there exist several effective methods to compute this part of the spectrum, e.g. by a discretization of either the time integration operator or the infinitesimal generator associated with the delay system [54 58]. Since the stability properties of the controlled system are determined by the characteristic roots with the leading real part, it is enough to employ a simple root-finding numerical algorithm based on the Newton-Raphson iteration method with a suitable chosen grid of starting values. For this purpose, we first make an implicit plot of the real and the imaginary parts of the characteristic Eq. (10) in the complex $\lambda$ plane to visualize the approximate location of the roots as intersecting points between the corresponding curves. In this way we obtain a coarse estimate of the location of the rightmost eigenvalues, the knowledge of which is then used to choose an appropriate grid of starting values encompassing this location.

By numerically solving Eq. (10) with the aforementioned procedure, we obtain the domains of successful control in the parameter plane $\left(K, T_{2}\right)$ for a fixed delay $T_{1}$ and for different values of the amplitude $\varepsilon$. The results are shown in Figs. 3 and 4. In the numerical calculations, we choose $T_{1}=23$ for which the original system is in a chaotic regime (see panel (d) in Fig. 2), having a positive value of the largest Lyapunov exponent $(\mathrm{LLE}=0.00973)$ 59]. The shaded areas (color online) correspond to the set of control parameters $\left(K, T_{2}\right)$ for which the maximum of the real part of the characteristic eigenvalues $\left\{\lambda_{i}\right\}$ is negative $\left(\max \left[\operatorname{Re}\left\{\lambda_{i}\right\}\right]<0\right)$, rendering the control successful. The values of $\max \left[\operatorname{Re}\left\{\lambda_{i}\right\}\right]$ are given by the grayscale (colorscale online) on the right in each figure, and the control is more robust as $\max \left[\operatorname{Re}\left\{\lambda_{i}\right\}\right]$ is more negative. The stability islands are surrounded by a "sea" of instability represented by the white region, for which the real part of the leading characteristic eigenvalue is positive $\left(\max \left[\operatorname{Re}\left\{\lambda_{i}\right\}\right]>0\right)$. The "coastline" between stability and instability (the stability border) is given in a parametric form via Eqs. (29) -(30) for $\varepsilon>0$ (VDFC), and via Eqs. (43)-(44) for $\varepsilon=0$ (TDFC). Panels (a) through (d) of Fig. 3 correspond to the modulation of the feedback delay $\tau(t)$ in a form of a sawtooth-wave, with amplitude values $\varepsilon=0,0.5,1$ and 2 , respectively. 

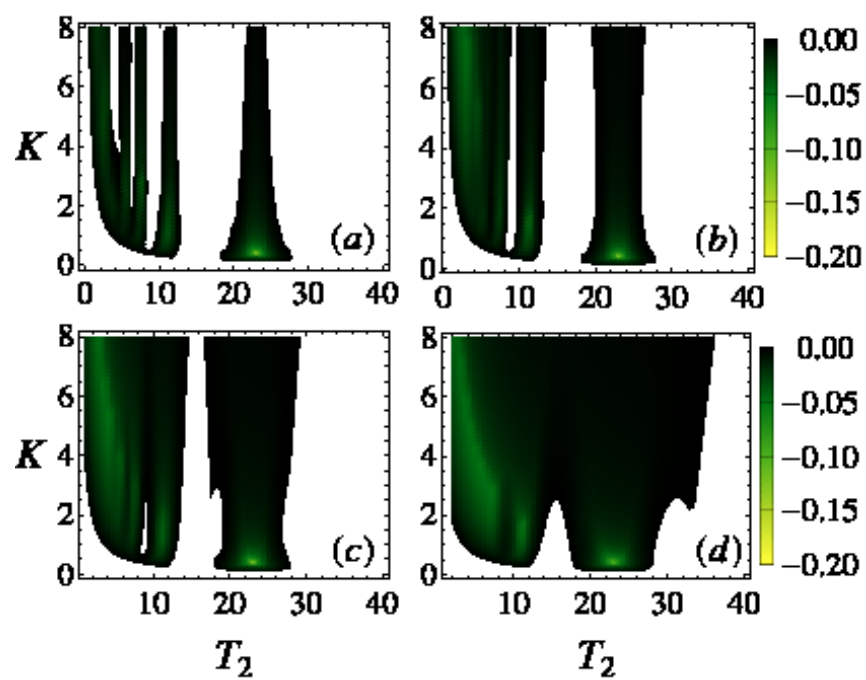

FIG. 3: (Color online) Domains of successful VDFC control in the $\left(K, T_{2}\right)$ plane for the unstable equilibria $x_{2,3}^{*}= \pm 1$ in the chaotic Mackey-Glass system $\left(T_{1}=23\right)$. The control delay $\tau(t)$ is modulated with a sawtooth-wave, and the values of the modulation amplitudes are: (a) $\varepsilon=0$ (TDFC); (b) $\varepsilon=0.5$, (c) $\varepsilon=1$; (d) $\varepsilon=2$. Combinations of $K$ and $T_{2}$ where VDFC successfully stabilizes the fixed points $x_{2,3}^{*}= \pm 1$ are plotted in graytones (colortones online). Note the shifts of the origin along the $T_{2}$ axes by an amount equal to $\varepsilon$ due to the limitation $T_{2} \geq \varepsilon$.

Panel (a) reveals the structure of the stability domain for $\varepsilon=0$ (TDFC). For the current choice of $T_{1}$, and also in general, there exists a stability region for relatively small $T_{1}$ with a complex structure, and a resonance island encompassing $T_{2}=T_{1}=23$ for which the control is most robust and can be achieved with smaller values of $K$. As $\varepsilon$ becomes larger than zero (VDFC, panels (b) $-(\mathrm{d}))$, the structure of the stability domain is reconfigured, resulting in a significant enlargement of the area of successful control. This enlargement is also observed for other delay modulations. In panels (a)-(b) of Fig. 4 we show the calculated stability domains for a sine-wave modulation for $\varepsilon=1$ and 2 , respectively, and (c)-(d) are the corresponding panels for a square-wave modulation. We note that for larger values of $\varepsilon$ in the case of a squarewave modulation, the stability area eventually spreads into several clearly distinguished stability islands, whose position is changing in an oscillatory manner as $\varepsilon$ further increases.

In Fig. 5 we show the stability domains in $\left(T_{1}, T_{2}\right)$ plane, fixing the feedback gain value at $K=0.5$. Panel (a) depicts the case when the modulation is absent (TDFC, $\varepsilon=0$ ), and panels (b)-(d) are related to sawtooth, sine and square-wave modulations, respectively, with $\varepsilon=2$. The diagrams show the typical enlargement of the stability area for VDFC with respect to TDFC. The parametric representation of the stability boundary is given by Eqs. (34)- (35).

To verify the analysis in the previous paragraphs, we
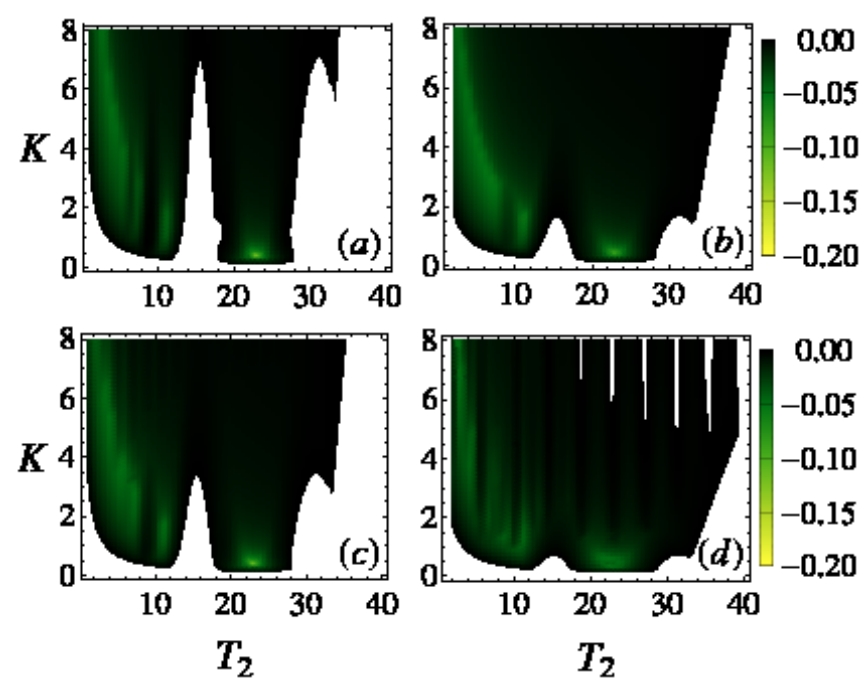

FIG. 4: (Color online) (a), (b) Stability domains of $x_{2,3}^{*}= \pm 1$ in the $\left(K, T_{2}\right)$ plane for the VDFC-controlled Mackey-Glass system with $T_{1}=23$. The delay modulation is in a form of a sine-wave with $\varepsilon=1$ (panel a) and $\varepsilon=2$ (panel b). (c), (d) Corresponding stability domains for a square-wave modulation. Note the shifts of the origin along the $T_{2}$ axes by an amount equal to $\varepsilon$ due to the limitation $T_{2} \geq \varepsilon$.


FIG. 5: (Color online) Domains of successful control in the $\left(T_{1}, T_{2}\right)$ plane for the unstable fixed points $x_{2,3}^{*}= \pm 1$ in the Mackey-Glass system. The feedback gain is fixed at $K=0.5$. (a) Stability diagram for $\varepsilon=0$ (TDFC). (b)-(d) Respective stability diagrams for sawtooth, sine and square-wave modulations with $\varepsilon=2$ (VDFC). Note that the minimum value of the $T_{2}$-axis in panels (b)-(d) is $T_{2}=2$ due to the limitation $T_{2} \geq \varepsilon$.

performed a computer simulation of VDFC for the fixed points $x_{2,3}^{*}= \pm 1$ by numerically integrating the system (46) for different delay modulations. The results are shown in Fig. 6. Panels (a), (c) and (e) depict the dynamics of the variable $x(t)$ for sawtooth, sine and squarewave modulations, respectively, and panels (b), (d) and 

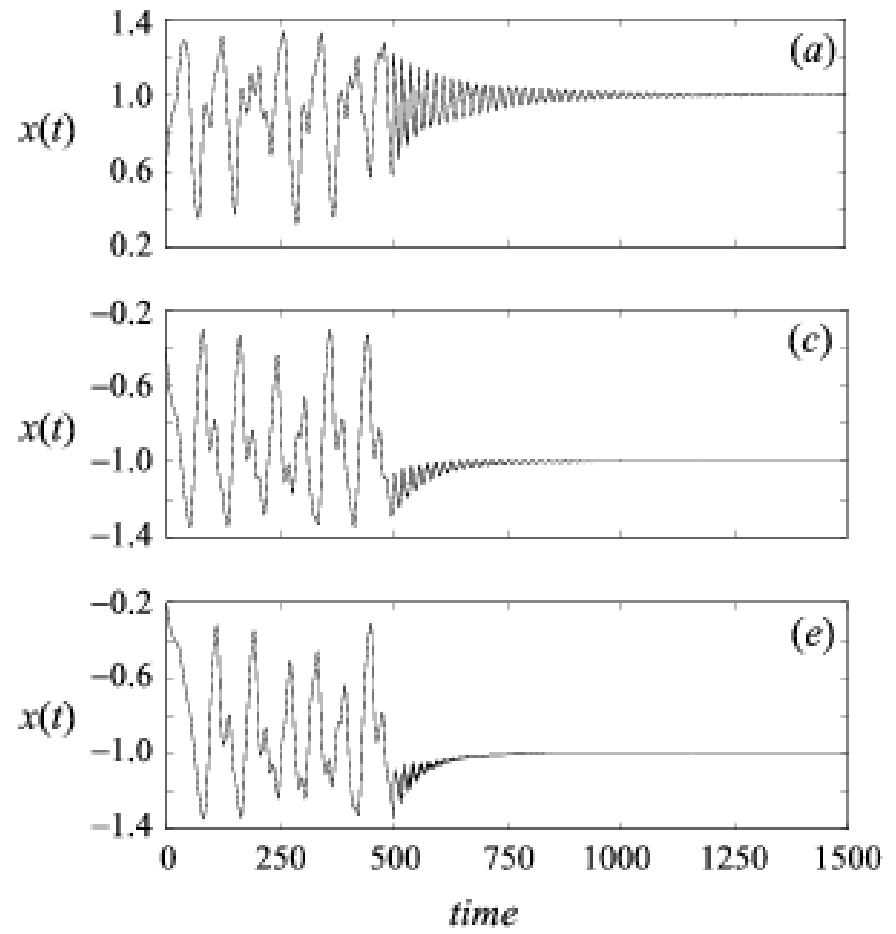


FIG. 6: VDFC applied to the chaotic Mackey-Glass system using different modulations of the delay-time $\tau(t)$. The parameters of the uncontrolled system are: $a=0.2, b=0.1, c=10, T_{1}=23$. (a), (b) Time plots of the variable $x(t)$ and the feedback signal $u(t)$ for a sawtooth-wave modulation, indicating a successful control of the unstable fixed point at $x_{2}^{*}=+1$. (c), (d) Stabilization of the unstable equilibrium at $x_{3}^{*}=-1$ with a sine-wave modulation. (e), (f) Time-series for a square-wave modulation stabilizing the unstable point at $x_{3}^{*}=-1$. In each case, the control parameters were: $K=2, \varepsilon=2, T_{2}=18$ and $\nu=5$. The control was activated at $t=500$. The total time span shown in each panel is 1500 time units. The simulations were performed using the MATLAB routine ddesd for integrating delay-differential equations with general delays.

(f) show the corresponding time-series of the feedback signal $u(t)$. In each case, the control parameters were chosen as $K=2, \varepsilon=2, T_{2}=18$ and $\nu=5$, fixing the delay of the uncontrolled system at $T_{1}=23$ for which the system is chaotic. We note that for these parameter values, the control via TDFC $(\varepsilon=0)$ is unsuccessful for any $K$, as can be perceived from the stability domain depicted in panel (a) of Fig. 3. Also, since $x_{2,3}^{*}$ have identical set of characteristic eigenvalues, they share common domains of successful control. However, they have different basins of attraction, and the preference of control towards either $x_{2}^{*}$ or $x_{3}^{*}$ depends on the initial conditions. In panels (b), (d) and (f) we see that the feedback signal $u(t)$ vanishes when the stabilization of the fixed point is achieved, suggesting noninvasiveness of VDFC, which is a consequence of the form of the control force in Eq. (4), since $x(t-\tau(t))=x(t)$ if the fixed point is stabilized.

When the frequency $\nu$ of the delay modulation is below the threshold (low-frequency modulation), the approximation of the variable-delay system with a distributeddelay system is not covered by Theorem A1, and, hence, the control domains cannot be calculated from the characteristic Eq. (10). However, the stability domains in this case can be obtained by numerically integrating the linear variable-delay system (7)-(8) for different values of the corresponding control parameters. In Fig. 7 we show the results of such a simulation in the parametric plane $\left(K, T_{2}\right)$ for $T_{1}=23$ and $\varepsilon=2$, taking the time-modulation of $\tau(t)$ in a form of a sawtooth wave. Different panels of the figure correspond to different values of the delay frequency $\nu$ : (a) $\nu=1.4$, (b) $\nu=1.8$, (c) $\nu=1.9$, (d) $\nu=2.0$, (e) $\nu=2.2$, (f) $\nu=2.4$, (g) $\nu=2.6$, (h) $\nu=3.0$. The combinations $\left(K, T_{2}\right)$ leading to a successful stabilization of the unstable fixed points $x_{2,3}^{*}= \pm 1$ are marked in black. It is observed that when the modulation frequency is about $\nu=3.0$ (panel (h)), the structure of the stability domain fairly resembles the stability domain for a high- $\nu$ modulations obtained from the characteristic Eq. (10) (compare with panel (d) in Fig. 3, noting the different scales on the $T_{2}$ axis). As expected, the simulations show that this resemblance becomes improved as $\nu$ attains higher values. On the other hand, the structure of the stability domain is gradually changing as $\nu$ becomes smaller than $\nu=3.0$ (panels (a)(g)), resulting in a reconstruction of the main domain and a birth of many small stability islands, clearly notable for larger nominal delays $T_{2}$ and approximately centered about those $T_{2}$ which are odd multiples of $\pi / \nu$. The emergence of this additional domain structure could be due to a resonance between the delay frequency $\nu$ and the intrinsic frequencies of the uncontrolled system, which are infinite in number. The distance between these res- 
onance islands $(\approx 2 \pi / \nu)$ becomes wider as $\nu$ decreases, and they become less pronounced for lower values of $\nu$. It can be noticed that the appearance of these resonance islands allows stabilization of the unstable equilibria for much larger nominal delays $T_{2}$ in comparison to the values of $T_{2}$ for a high- $\nu$ modulation. The simulations show that the range of the delay frequency parameter containing these resonance islands is strongly dependent on the system parameters (e.g., the modulation amplitude) and on the type of the delay modulation, and that this range of $\nu$ may not be continuous as in the current case, but it may consist of several different subintervals spread throughout the entire $\nu$-interval below some sufficiently high frequency and encompassing some of the values of $\nu$ coinciding with the eigenfrequencies of the uncontrolled system (see Fig. 8).

To check if the limitation of the control method asserted by Theorem 2 remains valid for low-frequency modulations, we have performed numerical simulations to determine the domains of successful VDFC control of the unstable equilibrium $x_{1}^{*}=0$, which has been shown uncontrollable via Theorem 2 for high-frequency modulations. The simulations in this case show the absence of the control domains in the corresponding parametric planes, suggesting the validity of Theorem 2 in the entire frequency range.

\section{CONCLUSION}

In conclusion, we have shown that variable-delay feedback control allows stabilization of unstable steady states in a class of scalar retarded time-delayed systems, represented by the chaotic Mackey-Glass system, over much larger domain of parameters in comparison to the usual Pyragas' delayed feedback control scheme. The analysis showed that the enlargement of the control domain may undergo a complex rearangement depending on the type and the frequency of the delay modulation. It is noticed that the enlargement of the control domain for highfrequency modulation of the delay is more pronounced when the variable delay is a continuous function of time in contrast to the case of variable delay function with a discontinuity leading to complex stability domain structure of a lesser magnitude. In the case of low-frequency modulation of the delay, we notice a complex rearrangement of the control domain, resulting in an appearance of extra stability islands, probably a consequence of a resonance between the frequency of the variable delay and the eigenfrequencies of the uncontrolled system. This resonance effect allows successful stabilization of the unstable fixed point for much larger nominal delays with respect to the situation when the frequency of the delay variation is above the threshold.

Limitation imposed by Theorem 2 shows that VDFC method fails to control certain unstable steady states for any value of the feedback control parameters in the case when the frequency of the delay modulation is high.
Moreover, numerical simulations suggest that this limitation is also valid for low-frequency modulations. Nevertheless, in lack of any analytical tool to treat a lowfrequency modulated VDFC, any general statement concerning the generalization of Theorem 2 to the whole frequency range should be taken cautiously, as well as the related observations concerning the aforementioned resonance phenomenon.

Putting the observations related to low-frequency modulation of the control delay on a firm mathematical basis constitutes an interesting subject for a future study. Other possible directions for future consideration would be stabilization of unstable steady states by VDFC in other types of DDE systems (e.g. systems described by neutral delay-differential equations [43]), in systems described by partial differential equations, and, also, implementation of the control method to stabilize unstable periodic orbits by a suitable choice of the delay modulation in order for the control method to stay noninvasive. An example for such a modulation in the latter case would be a periodic change of the control delay between $T$ and $2 T$, where $T$ is the period of the orbit to be stabilized 38.

\section{Acknowledgments}

We thank one of the referees for valuable comments that improve the presentation in the paper, in particular the discussion related to the resonance phenomenon in low-frequency modulations. We also appreciate fruitful discussions with B. Fiedler on the limitations of the "oddnumber limitation".

\section{Appendix A}

The stability of a linear (or linearized) RDDE system with a fast-varying delay can be obtained by studying the roots of the characteristic equation of the related time-invariant distributed delay system. The correctness of this approach is guaranteed only if the frequency of variation of the delay is large compared to the system's dynamics. A precise formulation of these assertions constitutes the following theorem:

Theorem A.1 Consider the linear system of variable delay differential equations:

$$
\begin{aligned}
\frac{d}{d t} \mathbf{x}(t) & =\hat{\mathbf{A}} \cdot \mathbf{x}(t)+\hat{\mathbf{B}} \cdot \mathbf{x}\left(t-T_{1}\right)+\mathbf{u}(t), \\
\mathbf{u}(t) & =\hat{\mathbf{K}} \cdot(\mathbf{x}(t-T(t))-\mathbf{x}(t)) \\
T(t) & =T_{2}+\varepsilon f(\nu t)
\end{aligned}
$$

where $\hat{\mathbf{A}}, \hat{\mathbf{B}}, \hat{\mathbf{K}} \in \mathbb{R}^{N \times N}$ are constant matrices, $\mathbf{x}(t) \in$ $\mathbb{R}^{1 \times N}$, and $f: \mathbb{R} \rightarrow[-1,1]$ is a periodic function with zero mean and period $2 \pi, \max f=1$, and $\min f=-1$. 

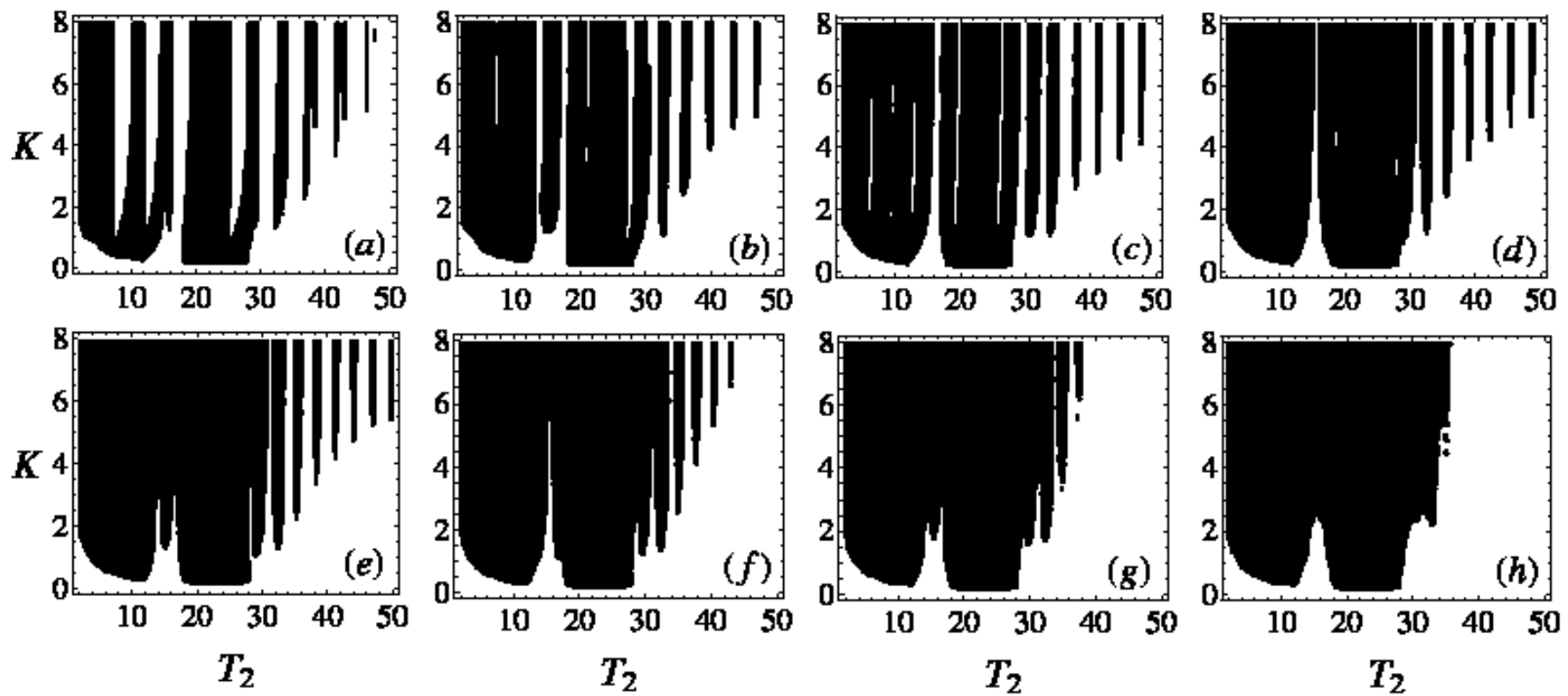

FIG. 7: The stability domains in $\left(K, T_{2}\right)$ plane related to the unstable steady states $x_{2,3}^{*}= \pm 1$ of the chaotic Mackey-Glass system $\left(T_{1}=23\right)$ for a low frequency modulation of the time delay $\tau(t)$. The delay modulation is in a form of a sawtooth-wave with $\varepsilon=2$, and the value of the modulation frequency is: (a) $\nu=1.4$, (b) $\nu=1.8$, (c) $\nu=1.9$, (d) $\nu=2.0$, (e) $\nu=2.2$, (f) $\nu=2.4$, (g) $\nu=2.6$, (h) $\nu=3.0$. The combinations $\left(K, T_{2}\right)$ leading to a successful stabilization of the unstable equilibria $x_{2,3}^{*}= \pm 1$ are marked in black. The characteristic eigenfrequencies of the uncontrolled system that lie in this interval of $\nu$ are: $1.43,1.71,1.98,2.25,2.52$ and 2.80 . Note the appearance of the resonance islands at the right of the main structure as $\nu$ becomes smaller than $\nu=3.0$. Also note the shifts of the origin along the $T_{2}$ axes by an amount equal to $\varepsilon$ due to the limitation $T_{2} \geq \varepsilon$
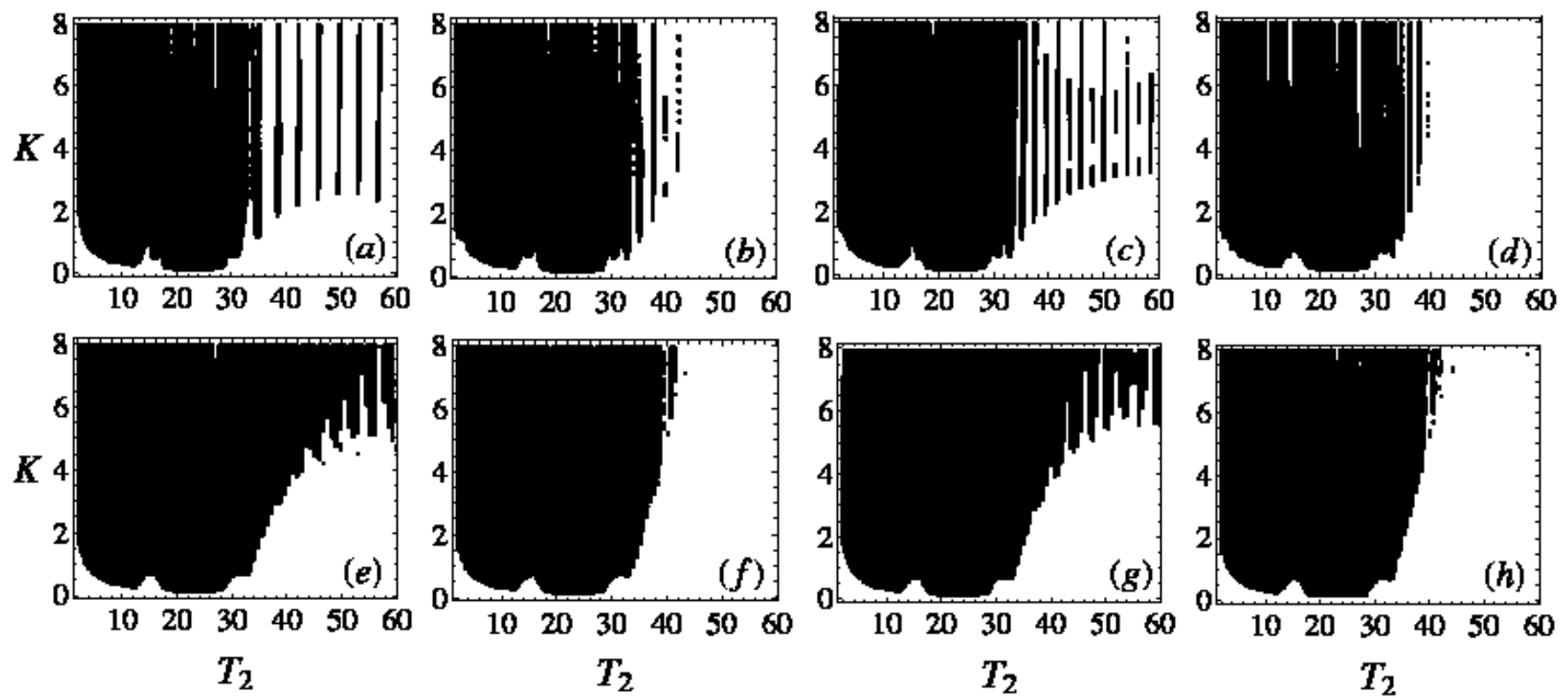

FIG. 8: A sample of the low-frequency control domains in $\left(K, T_{2}\right)$ plane corresponding to the unstable steady states $x_{2,3}^{*}= \pm 1$ of the chaotic Mackey-Glass system $\left(T_{1}=23\right)$ for a square-wave modulation with $\varepsilon=2$. The value of the modulation frequency is: (a) $\nu=1.7$, (b) $\nu=2.8$, (c) $\nu=3.0$, (d) $\nu=4.0$, (e) $\nu=8.0$, (f) $\nu=10.0$, (g) $\nu=11.0$, (h) $\nu=12.0$. The range of $\nu$ in which the resonance islands exist consists of several distinguished intervals encompassing some of the eigenfrequencies of the uncontrolled system: $1.71,3.07,7.99,10.99$. Note that although the modulation frequency $\nu$ in panel (b) coincides with one of the eigenfrequencies of the uncontrolled system $(\approx 2.8)$, the resonance islands are hardly noticeable in this case. 
Let $\varepsilon, T_{2}, \nu \in \mathbb{R}_{0}^{+}$, and $\varepsilon \leq T_{2}$. Let the integrable function $w:[-1,1] \rightarrow \mathbb{R}^{+}$be defined by:

$$
\int_{-1}^{1} \alpha(t) w(t) d t=\frac{1}{2 \pi} \int_{0}^{2 \pi} \alpha(f(t)) d t
$$

for every continuous function $\alpha:[-1,1] \rightarrow \mathbb{R}$. If the comparison system:

$$
\begin{aligned}
& \frac{d}{d t} \mathbf{x}(t)=\hat{\mathbf{A}} \cdot \mathbf{x}(t)+\hat{\mathbf{B}} \cdot \mathbf{x}\left(t-T_{1}\right)+ \\
&+\hat{\mathbf{K}} \cdot\left(\int_{t-T_{2}-\varepsilon}^{t-T_{2}+\varepsilon} \frac{w\left(\left(\theta-t+T_{2}\right) / \varepsilon\right)}{\varepsilon} \mathbf{x}(\theta) d \theta-\mathbf{x}(t)\right)
\end{aligned}
$$

is asymptotically stable, then the original system (A1)(A3) is globally uniformly asymptotically stable for large values of the frequency $\nu$ of the modulation.

Theorem A1 is a restatement of the main result in Ref. 46] to accomodate the present discussion [60], and its proof is based on an extension of the recently introduced trajectory-based proof technique [61]. According to Theorem A1, the stability of (A1) under the variable-delay control force (A2) can be inferred from the stablilty of the analogous time-invariant system (A5) with a distributed delay, for sufficiently large values of the parameter $\nu$ determining the frequency of the modulation. It is worth noting that Theorem A1 can be generalized to include the most general case of multiple delayed feedback terms in the control force $\mathrm{A} 2$ with different types of delay modulations 60]. The proof of this extension is straightforward, following the lines of the proof given in [46].

The comparison system (A5) can be recast in the form:

$$
\frac{d}{d t} \mathbf{x}(t)=\hat{\mathbf{A}} \cdot \mathbf{x}(t)+\hat{\mathbf{B}} \cdot \mathbf{x}\left(t-T_{1}\right)+\hat{\mathbf{K}} \cdot\left(\int_{-1}^{1} w(\eta) \mathbf{x}\left(\varepsilon \eta+t-T_{2}\right) d \eta-\mathbf{x}(t)\right),
$$

by making a change of the integration variable $\theta$ to the new variable $\eta$ through the relation $\theta=\varepsilon \eta+t-T_{2}$. Furthermore, by taking $\alpha(t)=1$ and $\alpha(t)=t$ in Eq. (A4) respectively, we obtain the relations involving the weight function $w$ :

$$
\begin{aligned}
& \int_{-1}^{1} w(t) d t=1 \\
& \int_{-1}^{1} t w(t) d t=0
\end{aligned}
$$

[1] K. Ogata, Modern control engineering (Prentice-Hall, New Jersey, 1997), third edition.

[2] E. Ott, C. Grebogy and J. A. Yorke, Phys. Rev. Lett. 64, 1196 (1990).

[3] E. Schöll and H. G. Schuster (ed.), Handbook of chaos control (Wiley-VCH, Weinheim, 2008), second completely revised and enlarged edition.

[4] S. Boccaletti, C. Grebogi, Y. C. Lai, H. Mancini and D. Maza, Phys. Rep. 329, 103 (2000).

[5] T. Kapitaniak (ed.), Controlling Chaos (Academic Press, London, 1996).

[6] F. T. Arecchi, S. Boccaletti, M. Ciofini and R. Meucci, Int. J. Bifur. Chaos 8, 1643 (1998).

[7] K. Pyragas, Phys. Lett. A 170, 421 (1992).

[8] K. Pyragas, Phil. Trans. R. Soc. A 364, 2309 (2006).

[9] P. Hövel and E. Schöll, Phys. Rev. E 72, 046203 (2005).

[10] S. Yanchuk, M. Wolfrum, P. Hövel and E. Schöll, Phys. Rev. E 74, 026201 (2006).

[11] T. Dahms, P. Hövel and E. Schöll, Phys. Rev. E 76,
From Eq. (A4), the weight $w$ can be interpreted as the probability distribution of $f(\xi)$, where $\xi$ is uniformly distributed over the interval $[0,2 \pi]$ (see Table I).
056201 (2007).

[12] K. Pyragas and A. Tamaševičius, Phys. Lett. A 180, 99 (1993).

[13] S. Bielawski, D. Derozier, and P. Glorieux, Phys. Rev. E 49, R971 (1994).

[14] T. Pierre, G. Bonhomme, and A. Atipo, Phys. Rev. Lett. 76, 2290 (1996).

[15] K. Hall, D. J. Christini, M. Tremblay, J. J. Collins, L. Glass, and J. Billette, Phys. Rev. Lett. 78, 4518 (1997).

[16] D.W. Sukow, M. E. Bleich, D. J. Gauthier, and J. E. S. Socolar, Chaos 7, 560 (1997).

[17] J. M. Krodkiewski and J. S. Faragher, J. Sound Vib. 234, 591 (2000).

[18] T. Fukuyama, H. Shirahama, and Y. Kawai, Phys. Plasmas 9, 4525 (2002).

[19] M. G. Rosenblum and A. S. Pikovsky, Phys. Rev. Lett. 92, 114102 (2004).

[20] C. von Loewenich, H. Benner, and W. Just, Phys. Rev. Lett. 93, 174101 (2004). 
[21] O. V. Popovych, C. Hauptmann, and P. A. Tass, Phys. Rev. Lett. 94, 164102 (2005).

[22] J. E. S. Socolar, D. W. Sukow and D. J. Gauthier, Phys. Rev E 50, 3245 (1994)

[23] K. Pyragas, Phys. Lett. A 206, 323 (1995).

[24] A. Ahlborn and U. Parlitz, Phys. Rev. Lett. 93, 264101 (2004).

[25] A. Ahlborn and U. Parlitz, Phys. Rev. E 72, 016206 (2005).

[26] H. G. Schuster and M. B. Stemmler, Phys. Rev. E 56, 6410 (1997).

[27] K. Pyragas, Phys. Rev. Lett. 86, 2265 (2001).

[28] K. Pyragas, V. Pyragas, I. Z. Kiss and J. L. Hudson, Phys. Rev. Lett. 89, 244103 (2002).

[29] K. Pyragas, V. Pyragas, I. Z. Kiss and J. L. Hudson, Phys. Rev. E 70, 026215 (2004).

[30] W. Just, T. Bernard, M. Ostheimer, E. Reibold and H. Benner, Phys. Rev. Lett. 78, 203 (1997).

[31] H. Nakajima, Phys. Lett. A 232, 207 (1997).

[32] H. Nakajima and Y. Ueda, Physica D 111, 143 (1998).

[33] B. Fiedler, V. Flunkert, M. Georgi, P. Hövel and E. Schöll, Phys. Rev. Lett 98, 114101 (2007).

[34] W. Just, B. Fiedler, M. Georgi, V. Flunkert, P. Hövel and E. Schöll, Phys. Rev. E 76, 026210 (2007).

[35] C. M. Postlethwaite and M. Silber, Phys. Rev. E 76, 056214 (2007).

[36] B. Fiedler, S. Yanchuk, V. Flunkert, P. Hövel, H.-J. Wünsche, and E. Schöll, Phys. Rev. E 77, 066207 (2008).

[37] A. Gjurchinovski and V. Urumov, Europhys. Lett. 84, 40013 (2008).

[38] A. Gjurchinovski and V. Urumov, in preparation.

[39] R. Bellmann and K. L. Cooke, Differential-Difference Equations (Academic Press, New York, 1963).

[40] J. K. Hale, Functional Differential Equations (Applied Mathematical Sciences Vol. 3, Springer, New York, 1971).

[41] A. Bellen and M. Zennaro, Numerical Methods for Delay Differential Equations (Clarendon Press, Oxford, 2003).
[42] M. Wolfrum and S. Yanchuk, Phys. Rev. Lett. 96, 220201 (2006).

[43] K. B. Blyuss, Y. N. Kyrychko, P. Hövel and E. Schöll, Eur. Phys. J. B 65, 571 (2008).

[44] T. Erneux, Applied Delay Differential Equations (Springer, New York, 2009).

[45] S. Yanchuk and P. Perlikowski, Phys. Rev. E 79, 046221 (2009).

[46] W. Michiels, V. Van Assche and S. Niculescu, IEEE Trans. Autom. Control 50, 493 (2005).

[47] P. Henrici, Applied and Computational Complex Analysis (Wiley, New York, 1974).

[48] K. Gu, S.-I. Niculescu and J. Chen, J. Math. Anal. Appl. 311, 231 (2005).

[49] M. C. Mackey and L. Glass, Science 197, 28 (1977).

[50] J. D. Farmer, Physica D 4, 366 (1982).

[51] P. Grassberger and I. Procaccia, Physica D 13, 34 (1984).

[52] A. Namajunas, K. Pyragas, and A. Tamaševičius, Phys. Lett. A 201, 42 (1995).

[53] A. Namajunas, K. Pyragas, and A. Tamaševičius, Phys. Lett. A 204, 255 (1995).

[54] D. Breda, Appl. Numer. Math. 56, 305 (2006).

[55] D. Breda, S. Maset and R. Vermiglio, IMA J. Numer. Anal. 24, 1 (2004).

[56] D. Breda, S. Maset and R. Vermiglio, SIAM J. Sci. Comput. 27, 482 (2005).

[57] K. Engelborghs and D. Roose, SIAM J. Numer. Anal. 40, 629 (2002).

[58] K. Verheyden, T. Luzyanina and D. Roose, J. Comp. Appl. Math. 214, 209 (2008).

[59] The spectra of Lyapunov exponents for RDDE systems were calculated using the algorithm in A. Wolf, J. B. Swift, H. L. Swinney and J. A. Vastano, Physica D 16, 285 (1985), following the idea suggested in Ref. [50].

[60] W. Michiels, private communication.

[61] L. Moreau and D. Aeyels, IEEE Trans. Autom. Control 45, 1554 (2000). 